\title{
A Comment or two on Holographic Dark Energy
}

\author{
A.J.M. Medved \\ Physics Department \\ University of Seoul \\ Seoul 130-743 \\ Korea \\ E-Mail(1): allan@physics.uos.ac.kr \\ E-Mail(2): joey_medved@yahoo.com
}

November 1, 2018

\begin{abstract}
It has, quite recently, become fashionable to study a certain class of holographicinspired models for the dark energy. These investigations have, indeed, managed to make some significant advances towards explaining the empirical data. Nonetheless, surprisingly little thought has been given to conceptual issues such as the composition and the very nature of the implicated energy source. In the current discourse, we attempt to fill this gap by the way of some speculative yet logically self-consistent arguments. Our construction takes us along a path that begins with an entanglement entropy and ends up at a Hubble-sized gas of exotic particles. Moreover, our interpretation of the dark energy turns out to be suggestive of a natural resolution to the cosmic-coincidence problem.
\end{abstract}




\section{Background and Buildup}

It is well acknowledged that our present-day universe is (or at least appears to be) in a phase of cosmological acceleration [1]. The simplest and (perhaps) most aesthetically pleasing explanation for this phenomenon would be a cosmological constant. (As was originally proposed by Einstein, albeit with a much different motivation in mind [2].) On the basis of Occam's Razor, this might well be the end of the story, except for a few points of notable infamy [3]:

(i) The cosmological-constant problem (version 1), or why is the empirically based value of the cosmological constant so small in comparison to the Planck scale (which is, as prescribed by quantum field theory, the natural scale that one would associate with the energy of the vacuum)? For future reference, the discrepancy between the two scales (empirical and Planck) is the staggering amount of at least 120 orders of magnitude.

(ii) The cosmological-constant problem (version 2), or given that the cosmological constant is so small, why is it not simply zero? To rephrase, suppose there is a fundamental symmetry at play (along the lines of supersymmetry) that is indeed responsible for Planckscale cancellations in the vacuum energy. Then what mechanism ends up breaking this symmetry at such an unnaturally small number $\left(10^{-120}\right)$ ?

(iii) The cosmic-coincidence problem, or why do we happen to live in the (cosmologically speaking) briefest of eras when the energy density of matter and the cosmological constant are virtually the same (i.e., within an order of magnitude)?

In view of a cosmological constant (per se) being somewhat problematic, many proposals have been suggested that supplant this fixed quantity with a more dynamical source. By current conventions, any such alternative (as well as the constant itself) falls under the generic classification of being a dark energy. 1 For instance, the potential energy for a slowly varying scalar field has often been nominated for the role of a dark energy; notably, such (so-called) quintessence models arise quite naturally out of the framework of string theory [7] and are analogous to the inflaton-description of inflation [8,9]. [The latter being the (generally accepted) earlier era of cosmic acceleration.]

There are, of course, a litany of dark energy proposals in the literature. Here, we will focus on what has become commonly known as the holographic model of dark energy. (See [10] for one of the seminal renditions, [11] for further discussion and [12] for an overview with almost too many references.) The basic premise is inspired by the famed holographic principle [13-16], with particular emphasis towards a pertinent observation made in [17]. We will now proceed to elaborate on this circle of ideas.

In spite of its many manifestations in the literature, the essence of the holographic principle is remarkably simple: a black hole represents, in a very fundamental sense, the maximally entropic object for a fixed amount of energy. As applied to a given (quantum) field theory, the principle advocates $S_{Q F T}<S_{B H}$ or, as long as our field theory comes equipped with an ultraviolet-energy cutoff $\Lambda$ and an infrared-length cutoff $L$, it would follow that

$$
L^{3} \Lambda^{3} \lesssim L^{2} M_{P}^{2}
$$

Here, we have only used the extensivity of the (unconstrained) field-theoretic entropy, the area-entropy law for black holes [18], and the (near) saturation of the initial inequality

\footnotetext{
${ }^{1}$ For further discussion on the cosmological-constant problems, various proposals for the dark energy, etcetera, see the reviews in $[4-6]$.
} 
at the respective cutoffs. 2

By very similar reasoning, one can talk about constraining the energy. In fact, by virtue of the hoop conjecture [19] (or, equivalently, the Schwarzschild limit), the field-theoretic energy should be limited by the linear size of the system. Then extensivity of the former and saturation at the cutoffs leads to

$$
L^{3} \Lambda^{4} \lesssim L M_{P}^{2}
$$

Since $L$ is, by hypothesis, the longest (meaningful) length scale in the theory, it follows that this second relation is the one that maximally constrains $\Lambda$. We are thus obliged to use the latter in obtaining the maximal energy density. Consequently,

$$
\rho \lesssim \Lambda^{4} \lesssim \frac{M_{P}^{2}}{L^{2}}
$$

It is this very last relation that served as the impetus for the notion of a "holographic dark energy". Namely, it has been suggested that the form of the dark energy should conform precisely with

$$
\rho_{D E} \leq c^{2} \frac{M_{P}^{2}}{L^{2}}
$$

where $c^{2}$ is a numerical factor which is always taken to be of the order of unity and can typically be constrained by the way of observational data. Predominantly (but not universally [20]), this has been regarded as a literal equality; meaning that the saturation point is to be chosen a priori.

What has never been particularly clear is to what choice should be made for the infrared cutoff $L$. The usual suspects are the apparent horizon or Hubble radius $H^{-1}=a / \dot{a}$, 3 the future (event) horizon and the past (particle) horizon. 4 Respectively, the latter two are as follows:

$$
\begin{aligned}
& d_{f}=a \int_{t}^{\infty} \frac{d t}{a}, \\
& d_{p}=a \int_{0}^{t} \frac{d t}{a} .
\end{aligned}
$$

At a first glance of the literature, it would appear that the future horizon is empirically favored [10] and the apparent horizon is emphatically ruled out [23]. Nevertheless, all bets remain on once the model is suitably complicated to include spatial curvature [24] and/or interactions with the matter sector [25,26], a time-varying gravitational coupling [25], the aforementioned non-saturated (holographic) bound [20,27], or whatever the flavor of the day happens to be. 5

\footnotetext{
${ }^{2}$ Further note that we choose, throughout, to work with a four-dimensional spacetime, set all fundamental constants - except for the Planck mass $M_{P}$ — to unity, and consistently ignore all inconsequential numerical factors.

${ }^{3}$ Here, $a=a(t)$ is the cosmological scale factor and a dot denotes a differentiation with respect to the cosmic time $t$. We will be presuming a flat universe in our discussions (hence, the equivalency of the apparent horizon and the Hubble radius), although almost nothing that is said really depends upon this distinction.

${ }^{4}$ This short list is by no means meant to be exhaustive. For instance, there is the "generalized" model of dark energy (for which $L$ is permitted to depend, functionally, on all three of the above) [21] and the "agegraphic" model of dark energy (whereby the age of the universe determines $L$ ) [22].

${ }^{5}$ Again, the reader should consult [12] for many other relevant citations.
} 
Irrespective of the exact choice for $L$, it is clear that the holographic model of dark energy has some attractive features. Firstly, it provides, by construction, an immediate (albeit somewhat ad hoc) solution to the cosmological-constant problem of the present era. Secondly, the fact that $L$ is (presumably) a dynamical quantity allows us to hope for even more: A fully realistic form of the model might be able to account for the dark energy in all other relevant eras (particularly, inflation) and accommodate a decelerating to accelerating phase (and vice versa) at the appropriate junctures. Thirdly, the holographic model has had, when suitably generalized (see the paragraph above), some degree of success in resolving even the cosmic-coincidence problem.

Nonetheless, there are (at least) a few aspects of the overall framework that remain puzzling. Clearly, this dark energy model is somewhat contrived; having been posed in such a manner so as to defer, rather than resolve, the very issues that inspired it. And let us ponder the following: What is the substance (for lack of a better word) that constitutes or underlies the implied dark energy? If the answer is some new type of particle, then where does this new entity fit into the grander scheme of things? (For instance, is it agreeable with the standard model?) If the answer is some manifestation of "pure geometry", then how is the use of the field-theoretic bounds to be justified in the first place? If the answer is none of the above, then are we really better off here than with a fixed cosmological constant?

Anyways, justifying and explaining the exact nature of the dark energy may be the least of our (conceptual) worries. The very definition of the holographic dark energy seems to hinge on the existence of a physically meaningful infrared cutoff; which is to say, a physically viable cosmological horizon. On the other hand, it is not at all evident that one can have such a horizon in the absence of some sort of dark energy. For instance, let us consider the popular choice of a future horizon. Without a dark energy or a cosmological constant to induce acceleration (and assuming no other forms of exotic matter are present), a simple calculation reveals that the future horizon is necessarily infinite. 6 Hence, there can be no such infrared cutoff without first having a dark energy and, likewise, there will be no holographic dark energy without having a cutoff to define it. This leaves us with a conceptual paradox that is tantamount to the proverbial question about "the chicken or the egg"!

Admittedly, it would be difficult to explain away any of these points until there is an established model of quantum gravity to call upon. So one might argue that any such issues should be deferred until that "messianic time" is finally upon us. But it might, just as well, be folly to continue tweaking a model that has no physically motivated rationalization other than appealing to a proposed principle of a yet unfounded theory.

On this last note, our current aim is to fill in the gap (somewhat) with a (n admittedly) speculative proposal for the nature of the holographic dark energy. Our starting point will be that the dark energy is induced from an entropy of entanglement, and the discussion will be advanced, sequentially, from there. As a way of motivation, an association between dark energy and entanglement appears to be a very natural one in a holographic context.

\footnotetext{
${ }^{6}$ In short, an accelerating universe and, thereby, finite future horizon requires an equation-of-state parameter (i.e., a ratio of pressure to energy density) of $\omega<-1 / 3$. [Alternatively, one can use the standard Friedmann result of $a \sim t^{2 / 3(1+\omega)}$ along with Eq.(5).] If the choices are limited to dust matter $(\omega=0)$, radiative matter $(\omega=1 / 3)$ and spatial curvature $(\omega=-1 / 3)$, it becomes quite evident that such an inequality is strictly unattainable. (For future reference, a "true" cosmological constant has $\omega=-1$ and the observational bound is $\omega<-0.75$ [1].)
} 
To elucidate, an entanglement entropy has been proposed $[28,29]$ as a viable explanation for the (holographic-inspiring) black hole area-entropy law. Moreover, it appears to have an important significance $[30,31]$ in the most rigorous realization of the holographic principle to date; namely, the $A d S-C F T$ correspondence [32]. [Note that the idea of connecting the dark energy to a quantum entanglement is not meant to be original: This connection was first proposed (concretely) in [33] and then advanced in [34,35]. Although there will inevitably be some overlap between the entanglement part of our discussion and these other works, we feel that the current perspective and interpretations are clearly distinct. 7]

The succession of points to be made will be organized as in the following summary: (1) Any relevant observer 8 will be causally separated from a significant portion of the universe. So, for physics to locally make sense, it is natural (if not absolutely necessary) to trace over these inaccessible degrees of freedom; thus giving rise to an entropy of entanglement.

(2) By some (yet-to-be-specified) process, the entanglement-induced degrees of freedom should be "uplifted" to the status of actual physical particles (insofar as the observer in question is concerned). Although unspecified, this process of uplifting can still be anticipated because of an analogy with the Unruh effect [37].

(3) Given the intuitively expected (and also substantiated $[38,39]$ ) form for an entanglement energy, it can (and will) be demonstrated that the energetics of the induced particles is in complete compliance with Eq.(44) for the (holographic) dark energy density.

(4) Although situated (at least in a holographic sense) at a causally defined horizon, the induced matter will be shown to be inherently non-localized; necessarily filling up the horizon interior in the guise of an inert gas of long-wavelength particles. In this way, it can be said that a space-filling dark energy has truly been achieved.

We will now proceed to elaborate on each of these points, in turn, and then conclude with an overview.

\section{The Main Points}

\section{$2.1 \quad$ Entanglement}

Thanks to the aftermath of inflation, the universe must be a vast, vast place; containing, at the very least, $e^{60}$ Hubble-sized spheres (and it could be substantially larger than this lower bound $[8,9]$ ). Meaning that, irrespective of the existence of a finite event (or some other formal) horizon, an observer will certainly be out of causal contact with a large fraction of the universe. [Given that inflation did occur, this must always be true except at very early (pre-inflationary) times and (depending on the overall energetics) asymptotically late times. Neither of these eras is of relevance to the current discussion.] The "rule-of-thumb" estimate for this "causal boundary" is a spherical surface of radius

\footnotetext{
${ }^{7}$ And, for the interested reader, see [36] for an altogether different viewpoint on the conceptual origin of the holographic dark energy.

${ }^{8}$ Whenever we refer to an "observer", it is meant to be in the most passive of senses. For instance, an observer might simply be a test particle that is being used to map out a specified world line in the spacetime. It should not (necessarily) be interpreted as an intelligent life form; even if our choice of semantics does imply this at times.
} 
$H^{-1}$, but the exact location need not concern us at this stage. 9 The important point is that, for all practical purposes, our observer's universe comes to an abrupt end at a distance of (roughly) one Hubble radius away.

If this discussion was limited to strictly classical physics, then this could well be the end of the story. But quantum considerations make for things to be significantly more interesting. Irregardless of the bounds of classical causality, it is safe to assume that most any matter or energy source that is outside of this casual barrier shares quantum correlations - or entanglements — with that found inside. (This follows from "EPRlike" quantum non-locality [41] and the realization that the entire universe was once a Planckian-sized place.) Following the standard ideas of quantum measurement [42], this means that, to have a sensible quantum description of the boundary interior, it is first necessary to trace over the degrees of freedom in the exterior.

More explicitly, let $\mid \Psi>$ represent the universal wavefunction, which is to be regarded as a pure (zero-entropy) state. Also, let $\left|\phi_{A}\right\rangle$ collectively denote a complete set of orthonormal states for the interior region and let $\mid \phi_{B}>$ do likewise for the exterior. It is known that one can always write (for a suitable choice of complex coefficients $C_{A B}$ )

$$
\left|\Psi>=\sum_{A} \sum_{B} C_{A B}\right| \phi_{A}>\mid \phi_{B}>
$$

Now, given the necessity to trace, the appropriate density matrix for an observer in the interior has the following reduced form:

$$
\begin{aligned}
\rho_{A} & =\operatorname{Tr}_{\mathrm{B}}[|\Psi><\Psi|] \\
& =\sum_{B} \sum_{A} \sum_{A^{\prime}} C_{A B} C_{A^{\prime} B}^{*}\left|\phi_{A}><\phi_{A^{\prime}}\right| .
\end{aligned}
$$

Let us recall that a gain in entropy can generally be associated with a loss in information. So that, on account of tracing out the exterior (an inherently information-negating process), the interior observer will naturally assign an entropy to her reduced subsystem. (Put differently, even though the initial state was pure, the reduced density matrix describes what is now a mixed state.) As should be well known, this entropy can be quantified with the use of the von Neumann formula

$$
S_{A}=-\operatorname{Tr}_{\mathrm{A}}\left[\rho_{A} \ln \rho_{A}\right] .
$$

As long as there are any quantum correlations at all between the two subsystems (i.e., as long as $\mid \Psi>$ can not be written as a direct product of the subsystems), it should be clear that this entanglement entropy will be a strictly positive quantity 10 — in spite of having started off with what was a pure state of (necessarily) vanishing entropy.

Let us next consider $S_{B}$; that is, the analogous entropy as would be assigned by an exterior observer after tracing over the interior states. After some minor manipulations, it should not be too difficult to convince oneself that $S_{B}=S_{A}$. This is, in actuality, generally true: Starting with a pure state in a region $V$, which is then split up into two

\footnotetext{
${ }^{9}$ For a best guess at a rigorous placement, we would suggest the "causal connection scale" as proposed in $[40]$.

${ }^{10}$ To see this, notice that $\rho_{A}$ is diagonal and, since all of the states have been properly normalized, each matrix entry is a non-negative number that does not exceed unity. The existence of correlations will mean at least one of these entries is non-vanishing.
} 
subregions - say $V_{A}$ and $V_{B}$ - one finds (after tracing) that this entropic equality is always preserved. The point is that this subdivision can be arbitrarily disproportionate; for instance, taking $V_{B} \gg V_{A}$, one would still find an equality between the corresponding entropies. (In fact, this is just the situation we would expect for the division between the boundary interior $A$ and exterior $B$.) This oddity would leave one to believe that the entanglement entropy can only depend on properties that are common to both subsystems. It would logically follow that, to leading order, the entanglement entropy should be proportional to the area of the surface that forms the common boundary between the regions. Reassuringly, this outcome has indeed been realized in previous (rigorously done) calculations $[28,29]$.

[As it now stands, the above line of reasoning is somewhat misleading. The existence of long-range correlations means that, generally speaking, the (leading-order) entanglement entropy is expected to scale with the volume of the total region [43] rather than just the (common) boundary area. As for the rigorous calculations, these apply to the special case of the quantum field theory being in its ground state. It, therefore, becomes prudent to ask if the ground state is a reasonable expectation in the current context. We would argue "yes!" on the premise that this particular entanglement is, in essence, a quantumgravitational process; and so the field theory should inherit an energy gap of (presumably) the order of $M_{P}$. Such a large gap would then act to suppress any excitations of the system out of its ground state and, thus, achieve the desired area scaling [44]. Alternatively, it has been shown [43] that the (holographic) energy bound of Eq.(2) is also a sufficient condition for the entanglement entropy to scale with the boundary area. Since we are, after all, talking about a holographic dark energy, it seems quite sensible that such a bound should (if necessary) be allowed to enter directly into the formalism as a principle of the fundamental theory.]

For our observer's Hubble-sized sphere in particular, it can now be safely anticipated that

$$
S_{E} \equiv S_{A \text { or } B} \sim H^{-2} M_{P}^{2}
$$

with the factor of $M_{P}^{2}$ following on dimensional grounds. This result will prove to be important a bit later on in the discussion. For now, the reader should keep in mind that the above entropy is an extremely large number; roughly, $10^{120}$ for the present-day value of the Hubble radius.

Before moving on, we would like to make another pertinent observation: Our notion of embedding all of the exterior physics into a single boundary surface is probably not much different from the principle of horizon complementarity [45]. 11] The latter prescribes, for an observer who is (necessarily) confined to a single Hubble-sized sphere in de Sitter space, that any other Hubble sphere provides a redundant description of the associated (quantum) Hilbert space — with this redundancy being holographically encoded in the encompassing horizon. Although the nature of the surfaces are somewhat different (causal boundary versus de Sitter horizon), there is clearly a common thread with regard to the storage of the exterior information.

\footnotetext{
${ }^{11}$ A topical note: Horizon complementarity can be viewed as a generalization of black hole complementarity [46], which is closely related, both conceptually and historically, to the holographic principle.
} 


\subsection{Uplifting}

For our argument to proceed forcefully onwards, it is necessary that the entanglement degrees of freedom be "uplifted" to the status of fully physical quanta. It is not entirely clear that such a claim could be valid, insofar as the entanglement in question is strictly an observer-dependent phenomenon. To avoid any confusion, we should point out that this concern is not at all an impediment to the entanglement interpretation of black hole entropy. In this case, the event horizon has a clear physical interpretation; irrespective of the presence or not of observers. To put this in another (metaphorical) way, if a black hole falls in a vacuum, it will "make a sound".

In spite of this observer-dependency sticking point, there are two credible reasons to believe in the physicality of this brand of entanglement. Firstly, the idea of a holographic inducement of physical matter is not without a precedent. For instance, various accounts of the (dynamical) brane-world model [47] would lead us to believe that some energy sources in the brane universe can be purely attributed to holographic inducement; vis-avis, the so-called mirage cosmologies [48]. Of further relevance, the AdS-CFT picture of holography [32] emphatically preaches that a quantum effect from one perspective can be classical from another, a string can be open and closed at the same time, a regime of strong coupling can just as easily be one of weak, the ultraviolet limit can be flipped around into the infrared, and so forth. There is certainly a lesson to be learned: Holographic dualities, when invoked, can distort (and even reverse!) the very essence of what might have been perceived as objective physicality. So given all this, it may not be unreasonable to expect that an observer-dependent effect as viewed from one side of a duality could still be fully physical from the other.

Secondly, and much less esoterically, one should be able to recognize a clear analogy between the present consideration and the Unruh effect [37]. To review, an observer in (flat) Minkowski space will, upon accelerating, perceive herself as being immersed in a bath of thermal radiation. 12 This is clearly an observer-dependent effect (no observer means no radiation), so is the perceived thermal bath real or not? Here, we know that the answer is a resounding yes! If the accelerating observer happens to be a particle detector - that is, the famous Unruh detector - then particles will be registered [49], even though the global picture is still a trivially flat spacetime.

So the moral of the story is that observer dependence need not be any impediment to physicality. But, then again, should we be bothered if the dark energy (physical or not) is an observer-dependent phenomenon? Here, the answer is no unless we can find a way to communicate with observers that can contradict our findings. Given the very definition of the causal boundary, such a communication is, by construction, outside the realm of possibility.

With the above arguments in tow, we feel ethically justified in assuming (albeit tentatively 13 ) that such an uplifting — from geometric entanglement to real particles can occur and will proceed accordingly. Now, with the uplifting taken to be in play, it is worth reflecting upon the status of (what we have been calling) the causal boundary.

\footnotetext{
${ }^{12}$ Note that the same observer would measure a negative value for the vacuum energy (that is, negative relative to the Minkowski vacuum), so there is no violation of energy conservation going on here.

${ }^{13}$ Ultimately, only the "true" theory of quantum gravity would be able to dispute or assert some of our claims. Nonetheless, for an article that does advocate the physicality of observer-dependent phenomena (at least in the analogous context of acceleration horizons), see [50].
} 
If these holographically induced particles are sufficiently energetic (as will be verified below), then what was once essentially a fictitious horizon can become quite real. That is to say, the presence of a dark-energy source (here, in the guise of the induced particles) could well be sufficient to create a future event horizon. [Besides the energetics, it is also necessary that the induced matter source has a negative enough pressure $(c f$, Footnote 6). On this point, we can only speculate on precisely what breed of particles would be suitable for this purpose. Suffice it to say, we must be talking about some highly exotic type of matter, which is a natural stipulation for any discussion on what would constitute the dark energy.]

Assuming that a future horizon is indeed realized, we now have an intriguing resolution to the "chicken-or-the-egg" conundrum that was raised above: On the one hand, the causality barrier induces an entanglement which then (by hypothesis) invokes the creation of real particles. On the other hand, these same particles uplift the status of this causal surface to a fully fledged horizon. In the sense that this holographic surface now has attained a clear physical relevance, it adds credence to the picture of the particles having, themselves, a real physical nature. What we have just described is, in essence, a quantumgravitational "bootstrap" process; whereby the horizon and particles are able to pull, concurrently, one another out from a sea of virtuality.

Finally, there is another point that should be emphasized. From our perspective, the relevant infrared cutoff is (essentially) the Hubble radius before the dark energy has been induced. 14 Which is to say, the dark energy does not predetermine the infrared cutoff; it is, if anything, the other way around. Hence, in our picture, the infrared cutoff can be expected to asymptotically go to infinity as the energy density of the "regular" matter dilutes to nothingness. (As an aside, such an evolution can be viewed as a renormalization group flow 15 to the infrared, with the cosmic-time coordinate serving as the scaling parameter.) This paints a very different picture from many other discussions on a holographic dark energy: Typically, the dark energy will ultimately dominate the evolution of the universe and continue to do so for the rest of time (e.g., [10]). Conversely, our interpretation is a dark energy that is never able to dominate over nor be dominated by the other sources of matter. 16 What is most significant about our viewpoint is the following: The cosmic-coincidence problem (why are the matter and dark energy of the same order in this particular era 17 ) would intrinsically be resolved!

One last aside: In light of the above discussion, this horizon can not necessarily be regarded as an event horizon per se. Perhaps it should, rather, be interpreted as an apparent horizon that behaves like an event horizon over sufficiently short time scales $\left(t \lesssim H^{-1}\right)$. Be that as it may, the horizon in question would, nevertheless, be a physically relevant one.

\footnotetext{
${ }^{14}$ By using the word "before", we do not mean to imply that there is a chronological order to this process. "Before" is meant, rather, in its logically precedent sense. Meanwhile, the relative timing of the events in this framework will be touched upon in the final subsection.

${ }^{15}$ Holographic manifestations of a renormalization group flow are not uncommon. See, for instance, [51].

${ }^{16} \mathrm{An}$ immediate exception could be in a very strongly gravitating cosmology for which the Hubble parameter is rapidly changing. In such a regime, the Hubble radius is no longer a reliable measure of causality [40]. Anyways, unless our universe is closed, this caveat has nothing to do with the future evolution.

${ }^{17}$ It is probably worth pointing out that, as the cosmos expand, the dust matter dilutes as $a^{-3}$ whereas a true cosmological constant would be unaffected. This is the discrepancy that makes the "coincidence" so disturbing.
} 


\subsection{Energetics}

As alluded to above, the next step will be to assess the energy density of these entanglementinspired particles. An agreement with Eq.(41) - that is, the originally proposed (holographic) dark energy density - can be viewed as an important self-consistency check of our framework. Conversely, a failure to do so would indicate that a conceptual retooling is called for.

The "energy of entanglement" is not as well understood as its entropic counterpart; however, the very same argument that brought us up to Eq.(10) can be expected to remain basically intact; namely, the entanglement energy should only depend on properties that are shared by the subdivided systems. Meaning that one would, once again, intuitively expect the leading-order contribution to go as the area of the common boundary surface. As before, rigorous calculations do bear this intuitive reasoning out [38, 39]. 18 This deduction along with dimensional considerations dictates an entanglement energy (for the interior or exterior) of

$$
E_{E} \sim H^{-2} M_{P}^{3}
$$

After dividing this energy by the interior volume $\left(\mathrm{H}^{-3}\right)$, one might suspect that there is already a problem with the proposal. However, as we will now demonstrate, this is actually not the case. What has just been deduced is the energy as it would be measured locally at the horizon surface. Meanwhile, what we should really be examining is the observationally relevant energy; that is, the energy as measured by the observer whose sphere of causality defines the horizon. Now it becomes especially pertinent to the discussion that the causal boundary has, indeed, been elevated to a physical horizon - as would be expected by the presence of a legitimate dark energy source. Having an (apparent) event horizon means that we are really talking about an asymptotically de Sitter spacetime.

To proceed in a quantitative fashion, we will require a coordinate system. For the purposes of our "ball-park" estimates, it will be sufficient to employ a suitably chosen set of de Sitter coordinates. Then the best choice, as relevant to an observer (at the origin) with no access to the horizon exterior, is the well-known static patch of de Sitter space [52]:

$$
d s^{2}=-\left[1-\frac{r^{2}}{H^{-2}}\right] d \tau^{2}+\left[1-\frac{r^{2}}{H^{-2}}\right]^{-1} d r^{2}+r^{2} d \Omega_{2}^{2} .
$$

Note that the horizon is located at $r=H^{-1}$.

What is most important, for the current evaluation, is the effect of the gravitationally induced redshift [53]. For an observer (at the origin) viewing any other point in the interior, this effect will depend on the radial separation and can be quantified by the following "redshift factor":

$$
\mathcal{Z}(r) \equiv \sqrt{\frac{-\left.g_{\tau \tau}\right|_{r}}{-\left.g_{\tau \tau}\right|_{0}}}=\sqrt{1-\frac{r^{2}}{H^{-2}}} .
$$

\footnotetext{
${ }^{18}$ One caveat for the energy calculation is that the expectation value of the energy for the undivided spacetime should be parametrically smaller than the surface area of the common boundary. Since, in our case, the field theory is to be regarded as residing in its ground state [as per the parenthetic discourse just above Eq.(10]), it is quite doubtful that this technicality could be of any issue here.
} 
Near the horizon, this factor becomes

$$
\mathcal{Z}\left(r \lesssim H^{-1}\right) \sim \sqrt{\frac{1}{H^{-1}}\left(H^{-1}-r\right)}
$$

and, quite obviously, $\mathcal{Z}\left(r=H^{-1}\right)=0$.

Text-book discussions on gravitational redshifts tell us that $E_{O} \sim E_{E} \mathcal{Z}\left(H^{-1}\right)$, where $E_{O}$ is meant to denote the energy of observational relevance. Naively, the vanishing redshift factor implies that this energy also vanishes. However, by virtue of the (quantum) uncertainty principle, it is not reasonable to presume this degree of localization in the calculation. A better rationalized approach is to calculate the redshift at some ultravioletcutoff point. Following the methodology of the "brick-wall" calculations of black hole entropy [54] (also see [55,56]), we will judiciously place the cutoff at a proper distance of $M_{P}^{-1}$ away from the horizon. Recognizing that the proper radial distance is measured by $d r \sqrt{g_{r r}}$ and (near the horizon) $g_{r r}^{-1}=-g_{\tau \tau} \sim \mathcal{Z}^{2}$, we can then deduce that [also using $\left.x \equiv r / H^{-1} \lesssim 1\right]$

$$
\begin{aligned}
M_{P}^{-1} & \sim H^{-1} \int_{x}^{1} \frac{d x}{\mathcal{Z}} \sim H^{-1} \int_{x}^{1} \frac{d x}{\sqrt{1-x}} \\
& \sim H^{-1} \sqrt{1-x} \sim H^{-1} \mathcal{Z} .
\end{aligned}
$$

From the above, it follows that

$$
\mathcal{Z} \sim \sqrt{1-x} \sim H M_{P}^{-1}
$$

for the near-horizon redshift and then [with the help of Eq.(11)]

$$
E_{O} \sim \mathcal{Z} E_{E} \sim H^{-1} M_{P}^{2}
$$

for the observationally relevant energy. Dividing by the volume of the Hubble sphere, we now obtain an energy density of

$$
\rho_{O} \sim \frac{E_{O}}{H^{-3}} \sim H^{2} M_{P}^{2}
$$

With the obvious identification of $H^{-1}$ with the infrared cutoff $L$, this computation is in complete compliance with the holographic form of Eq.(4) 19

\subsection{Non-Locality}

One might be bothered by a dark energy that is strongly localized at a horizon. Indeed, such an energy source could very well be detectably different from the cosmological constant that it aspires to replicate. In our picture, the horizon certainly represents the surface of inducement (via entanglement), but a simple argument will reveal that the dark energy must necessarily delocalize and fill up the Hubble sphere.

Let us now explicate this point. To discuss the locality (or lack thereof) of a given particle, it is usually sufficient to consider its Compton wavelength $\lambda_{C}$. By recalling the

\footnotetext{
${ }^{19}$ To be clear, we are not saying here that $L$ should be unequivocally identified as the Hubble radius but, rather, that these length scales will be of the same order of magnitude. That is to say, we would not anticipate any conflict with observation in the sense suggested by [23].
} 
origin of the dark energy as being a process of entanglement, we can immediately constrain the associated $\lambda_{C}$ from above. Significantly, the entanglement procedure entailed tracing over all of the degrees of freedom to the exterior of (what is now) the horizon. Hence, insofar as it "concerns" a dark-energetic particle, the universe must end at the horizon; which is to say, there is, practically speaking, no longer an exterior region to speak of. And so, it must follow that

$$
\lambda_{C} \leq H^{-1}
$$

To constrain $\lambda_{C}$ from below, we need only to invoke the usual statistical interpretation of an entropy, along with the uncertainty principle. Applying the former input to Eq.(10), we count the number of particles to be (roughly) $N \sim H^{-2} M_{P}^{2}$; meaning that the energy per particle is $E_{O} / N \sim H$. As dictated by the uncertainty principle, the inverse of this ratio provides a lower bound for the spatial extent of any given particle, or

$$
\lambda_{C} \geq H^{-1}
$$

There is, obviously, only one way to avoid a contradiction between Eqs.(19) and (20); namely,

$$
\lambda_{C} \sim H^{-1} .
$$

And so, not only are the particles not localized at the horizon, they are delocalized across the full extent of the Hubble sphere!

The picture is now the following: We started with degrees of freedom being excited on a spherical surface, and then end up with a sphere-filling gas of extremely long-wavelength and inert 20 particles. Note that there is no need to establish a dynamical mechanism for this delocalization to happen. From a holographic vantage point, the cosmic-time coordinate is (as mentioned above) the scale factor of a renormalization group flow: Meaning that the flow of cosmic time coincides with the changes in the scale of the infrared cutoff $\left(L \sim H^{-1}\right)$ but has nothing to say about the occurrence of holographically triggered mechanisms. These take place in some abstract (from our perspective) realm which is governed by a time evolution that is - in all likelihood - unrelated to the flow of cosmic time. And so, cosmically speaking, the particles have always been there and are always delocalized in accordance with the scale set, at any given time, by $L=L(t)$. 21

Nevertheless, one would still be entitled to ask if there is a physical framework that supports such a dramatic degree of ubiquity. That is to say, is there any credible reason to believe in the existence of particles with such an unnaturally long wavelength? We will now proceed to argue that this type of delocalization is actually quite natural in the current context.

To begin here, let us ponder as to what might be the nature of these holographically induced particles. The key word here is holograhic(ally): The pertaining principle is often asserted to be a statement about - or even a manifestation of - quantum gravity. Alternatively, regarding this principle as an effect rather than a cause, one might prefer to focus on the entanglement origin of these particles and just leave holography out of it. But, even then, entanglement entropy can similarly be viewed as a synthesis of gravitational

\footnotetext{
${ }^{20}$ We say "inert" because these particles should, by default, be able to mimic a cosmological constant. At this point, the property of inertness must be put in by hand; however, see below.

${ }^{21}$ Let us point out, once again, that $L$ might be much different than $H^{-1}$ when the universe is strongly gravitating; in particular, in the earliest stages of the cosmological evolution.
} 
and quantum concepts; inasmuch as geometry (which underlies entanglement) is the very essence of gravity. So, by all accounts, these particles must, on some level, be identifiable as "quanta of geometry". 22

Continuing along this line of inquiry, what (if anything) can we say about such geometric quanta? Undoubtedly, the answer will be highly model dependent. However, it is notable that both string theory (especially in its matrix realization [57]) and loop quantum gravity [58] share something in common when informing us about their fundamental quanta; respectively, D0-branes and spin junctions. For both of these models, the fundamental constituents are expected to behave, collectively, as a gas of non-interacting and fully distinguishable particles [59-65]. This is a much different state of affairs from the "garden-variety" particles — bosons and fermions — which are (of course) always treated as being indistinguishable.

What is particularly interesting (for us anyways) about distinguishable particles is that they obey a generalized form of Bose and Fermi statistics that is known as infinite statistics [66]. More formally,

$$
a_{j} a_{k}^{\dagger}-q a_{k}^{\dagger} a_{j}=\delta_{j k}
$$

where $a_{j}$ and $a_{k}^{\dagger}$ represent the usual lowering and raising operators, while $q$ is a realnumber parameter that can range between -1 (the fermionic limiting case) and +1 (the bosonic limit). Thanks to the above symbolic convention, such particles are often referred to as quons.

Now we finally come to the punch line. Infinite statistics will inevitably describe a theory that is inherently non-local. To see this, it is sufficient to consider the number operator; which can be suitably defined by the commutator relation

$$
\left[N_{j}, a_{k}^{\dagger}\right]=\delta_{j k} a_{k}^{\dagger}
$$

Choosing the $q=0$ case for simplicity, one can readily be convinced that the number operator must take on the recursive form

$$
N_{j}=a_{j}^{\dagger} a_{j}+\sum_{k} a_{k}^{\dagger} a_{j}^{\dagger} a_{j} a_{k}+\sum_{l} \sum_{k} a_{l}^{\dagger} a_{k}^{\dagger} a_{j}^{\dagger} a_{j} a_{k} a_{l}+\ldots
$$

And so one finds that, even for the simplest $(q=0)$ case, the resulting theory is a non-local one, and the expansions are even more convoluted when $0<|q|<1$. 23

Let us tie this altogether. Our dark energy particles must be delocalized in accordance with Eq.(21). Meanwhile, whatever these particles exactly are (dubbed as quanta of geometry above), the consensus view of quantum-gravity theorists is that they should be fully distinguishable and, thereby, obey infinite statistics. Conveniently, infinite statistics can describe a non-local yet otherwise sensible quantum field theory. Meaning that, given the quantum-gravitational pedigree of the particles, this delocalization of the dark energy

\footnotetext{
${ }^{22}$ We have purposely avoided saying "quanta of gravity", which might be misconstrued as a synonym for gravitons.

${ }^{23} \mathrm{It}$ is worth pointing out that this non-locality does not jeopardize some of the more desirable features of conventional quantum theory such as the TCP theorem and cluster decomposition. So if non-locality is, in itself, not an issue, the quantum practitioner need not be overly concerned about harmful "side effects".
} 
turns out to be quite natural (and perhaps even to be anticipated). As an added bonus, the fundamental particles of quantum gravity are expected to be non-interacting, which is an almost essential stipulation for the (presumably) inert substance that would constitute the dark energy.

Before concluding, we would be remiss to not mention a pair of recent papers that have already made a connection between the dark energy and infinite statistics $[67,68]$. These studies certainly inspired a substantial amount of the current subsection, and we hope that our perspective was sufficiently different to contribute to the discussion.

\section{Overview}

The holographic model of dark energy [10] (including the many variations thereof [12]) has proven to be a promising avenue for understanding the current acceleration of the universe. As already reflected upon, this model has been demonstrated to have an ample amount of potential for matching the empirically based expectations of the observable universe; with regard to both the past and the present. What is still lacking, however, is a viable explanation of what the holographic dark energy is exactly supposed to represent. That is to say, what is the composition of the implicated energy source and why does this substance exhibit the properties that are desirable for mimicking a cosmological constant?

In the current paper, we have made an initial attempt at filling in this gap. Many of our arguments were admittedly speculative and many details were regrettably left out. Nonetheless, we still feel that some modest progress has been achieved along the stated lines. Our construction follows a logical succession of ideas with a self-consistent prediction for the energy density. Moreover, just by thinking about the issues "out loud", we have been able to make some deductions that have been previously missed or, at least, mostly overlooked. Most significantly, our discussion has cast a new light upon the relationship between the holographic dark energy and its associated infrared cutoff. From our perspective, the latter is an antecedent for the former; meaning that the magnitude of the dark energy is fixed to (more or less) match the other matter sources in the universe. If this turns out to be a valid assessment of the situation, then the (so-called) cosmiccoincidence problem would be quite naturally resolved.

Obviously, there is much more work to be done; either in addressing the outstanding questions of the current proposal or in constructing an altogether different framework that starts anew. It is hoped that both paths are enthusiastically followed; not only by the current author but by others in the field as well. 


\section{Acknowledgments}

Research is financially supported by the University of Seoul. The author thanks Yun Soo Myung for his inputs and valued discussions, and CQUeST at Sogang University for their hospitality. The author also thanks Stephen Hsu for pointing out an oversight in the first rendition of the manuscript.

\section{References}

[1] S. Perlmutter et al., Nature 391, 51 (1998).

[2] A. Einstein, Sitzungsber. Preuss. Akad. Wiss. phys.-math. Klasse VI, 142 (1917).

[3] S. Weinberg, Rev. Mod. Phys. 61, 1 (1989);

"The Cosmological Constant Problems", arXiv:astro-ph/0005265 (2000).

[4] S.M. Carroll, Living Rev. Rel. 4, 1 (2001) arXiv:astro-ph/0004075.

[5] P.J.E. Peebles and B. Ratra, Rev. Mod. Phys. 75, 559 (2003) arXiv:astro-ph/0207347.

[6] T. Padmanabhan, "Dark Energy and Gravity", arXiv:0705.2533 and to appear in Gen. Rel. Grav. (2007).

[7] C. Wetterich, Nucl. Phys. B 302, 668 (1988).

[8] A.A. Starobinsky, Phys. Lett. B 91, 99 (1980).

[9] A. Guth, Phys. Rev. D 23, 347 (1981).

[10] M. Li, Phys. Lett. B 603, 1 (2004) arXiv:hep-th/0403127.

[11] Y.S. Myung, Phys. Lett. B 610, 18 (2005) arXiv:hep-th/0412224].

[12] M. Li, C. Lin and Y. Wang, "Some Issues Concerning Holographic Dark Energy", arXiv:0801.1407 (2008).

[13] J.D. Bekenstein, Phys. Rev. D 23, 287 (1981).

[14] G. t Hooft, "Dimensional Reduction in Quantum Gravity", arXiv:gr-qc/9310026 (1993).

[15] L. Susskind, J. Math. Phys. 36, 6377 (1995) arXiv:hep-th/9409089].

[16] R. Bousso, Rev. Mod. Phys. 74, 825 (2002) arXiv:hep-th/0203101.

[17] A.G. Cohen, D.B. Kaplan and A.E. Nelson, Phys. Rev. Lett. 82, 4971 (1999) arXiv:hep-th/9803132.

[18] J.D. Bekenstein, Lett. Nuovo. Cim. 4, 737 (1972);

Phys. Rev. D 7, 2333 (1973); Phys. Rev. D 9, 3292 (1974). 
[19] K. S. Thorne, in Magic without Magic: John Archibald Wheeler, ed. J. Klauder (Freeman, San Francisco, 1972).

[20] D. Pavon and W. Zimdahl, Phys. Lett. B 628, 206 (2005) arXiv:gr-qc/0505020].

[21] S. Nojiri and S.D. Odintsov, Gen. Rel. Grav. 38, 1285 (2006) arXiv:hep-th/0506212.

[22] R.-G. Cai, Phys. Lett B 657228 (2007) arXiv:0707.4049].

[23] S.D.H. Hsu, Phys. Lett. B 594, 13 (2004) arXiv:hep-th/0403052].

[24] Q.-G. Huang and M. Li, JCAP 0408, 013 (2004) arXiv:astro-ph/0404229.

[25] R. Horvat, Phys. Rev. D 70, 087301 (2004) arXiv:astro-ph/0404204.

[26] W. Zimdahl and D. Pavon, "Interacting holographic dark energy", arXiv:astro-ph/0606555 (2006).

[27] B. Guberina, R. Horvat and H. Nikolic, Phys. Rev. D 72125011 (2005) arXiv:astro-ph/0507666.

[28] L. Bombelli, R. Koul, J. Lee and R. Sorkin, Phys. Rev. D 34, 373 (1986).

[29] M. Srednicki, Phys. Rev. D 71, 66 (1993).

[30] S. Ryu and T. Takayanagi, Phys. Rev. Lett. 96, 181602 (2006) arXiv:hep-th/0603001.

[31] D.V. Fursaev, JHEP 0609, 018 (2006) [arXiv:hep-th/0606184].

[32] J.M. Maldacena, Adv. Theor. Math. Phys. 2, 231 (1998) arXiv:hep-th/9711200.

[33] J.-W. Lee, J. Lee and H.-C. Kim, JCAP 08, 005 (2007) arXiv:hep-th/0701199.

[34] J.-W. Lee, J. Lee and H.-C. Kim, "Quantum Informational Dark Energy: Dark energy from forgetting", arXiv:0709.0047 (2007).

[35] R. Horvat, "Holographic dark energy: quantum correlations against thermodynamical description", arXiv:0711.4013 (2007).

[36] E.N. Saridakis, "Restoring Holographic Dark Energy in Brane Cosmology", arXiv:0712.2228 and to appear in Phys. Lett. B (2007).

[37] W.G. Unruh, Phys. Rev. D 14, 870 (1976).

[38] R. Brustein, D. Eichler, S. Foffa and D.H. Oaknin, Phys. Rev. D 65, 105013 (2002) arXiv:hep-th/0009063.

[39] R. Brustein and A. Yarom, JHEP 0501, 046 (2005) arXiv:hep-th/0302186.

[40] R. Brustein and G. Veneziano, Phys. Rev. Lett. 84, 5695 (2000) arXiv:hep-th/9912055.

[41] A. Einstein, B. Podolsky and N. Rosen, Phys. Rev. 47, 777 (1935). 
[42] N.J. Cerf and C. Adami, "Quantum Mechanics of Measurement", arXiv:quant-ph/9605002 (1996).

[43] R. Buniy and S. Hsu, Phys. Lett. B 644, 72 (2007) arXiv:hep-th/0510021.

[44] M. Requardt, "Entanglement-Entropy for Groundstates, Low-lying and Highly Excited Eigenstates of General (Lattice) Hamiltonians", arXiv:hep-th/0605142 (2006).

[45] T. Banks and W. Fischler, "M-Theory Observables for Cosmological Spacetimes", arXiv:hep-th/0102077 (2001).

[46] L. Susskind, L. Thorlacius and J. Uglum, Phys. Rev. D 48, 3743 (1993) arXiv:hep-th/9306069.

[47] P. Kraus, JHEP 9912, 011 (1999) arXiv:hep-th/9910149.

[48] A. Kehagias and E. Kiritsis, JHEP 9911, 022 (1999) arXiv:hep-th/9910174.

[49] N.D. Birrell and P.C.W. Davies, Quantum fields in curved space (Cambridge University Press, Cambridge, 1982).

[50] H. Culetu, Int. J. Mod. Phys. D 15, 2177 (2006) arXiv:hep-th/0607049.

[51] J. de Boer, E. Verlinde and H. Verlinde, JHEP 0008, 003 (2000) arXiv:hep-th/9912012.

[52] M. Spradlin, A. Strominger and A. Volovich, "Les Houches Lectures on De Sitter Space", arXiv:hep-th/0110007 (2001).

[53] R. Tolman and P. Ehrenfest, Phys. Rev. 36, 1761 (1930).

[54] G. t Hooft, Nucl. Phys. B 256, 727 (1985).

[55] S. Mukohyama, M. Seriu and H. Kodama, Phys. Rev. D 55, 7666 (1997) arXiv:gr-qc/9701059.

[56] Y.S. Myung, Phys. Lett. B 636, 324 (2006) arXiv:gr-qc/0511104.

[57] T. Banks, W. Fischler, S.H. Shenker and L. Susskind, Phys. Rev. D 55, 5112 (1997) arXiv:hep-th/9610043.

[58] A. Ashtekar, C. Rovelli and L. Smolin, Phys. Rev. Lett. 69, 237 (1992) arXiv:hep-th/9203079.

[59] A. Strominger, Phys. Rev. Lett. 71, 3397 (1993) arXiv:hep-th/9307059.

[60] I.V. Volovich "D-branes, Black Holes and $S U(\infty)$ Gauge Theory", arXiv:hep-th/9608137 (1996).

[61] T. Banks, W. Fischler, I.R. Klebanov and L. Susskind, JHEP 9801, 008 (1998) arXiv:hep-th/9711005.

[62] D. Minic, "Infinite Statistics and Black Holes in Matrix Theory", arXiv:hep-th/9712202 (1997). 
[63] K.V. Krasnov, Phys. Rev. D 55, 3505 (1997) arXiv:gr-qc/9603025.

[64] C. Rovelli, Phys. Rev. Lett. 77, 3288 (1996) arXiv:gr-qc/9603063.

[65] S.A. Major and K.L. Setter, Class. Quant. Grav. 18, 5125 (2001) arXiv:gr-qc/0101031.

[66] O.W. Greenberg, "Quons, an interpolation between bose and fermi oscillators", arXiv:cond-mat/9301002 (1993).

[67] Y.J. Ng, "Holographic Foam, Dark Energy and Infinite Statistics", arXiv:gr-qc/0703096 and to appear in Phys. Lett. B (2007).

[68] V. Jejjala, M. Kavic and D. Minic, "Fine Structure of Dark Energy and New Physics", arXiv:0705.4581 (2007). 